\title{
Bivariate Normal Distribution
}

National Cancer Institute

\section{Source}

National Cancer Institute. Bivariate Normal Distribution. NCI Thesaurus. Code C53217.

A normal distribution which models two variables of interest. The parameters of the bivariate normal distribution include the mean and variance of each individual variable and the correlations between variables. 